\title{
Investigating BSM Models with Large Scale Separation
}

\author{
Anna Hasenfratz ${ }^{1}$, Claudio Rebbi ${ }^{2, \star}$, and Oliver Witzel ${ }^{3 \star \star}$ \\ ${ }^{1}$ Department of Physics, University of Colorado, Boulder, CO 80309, USA \\ ${ }^{2}$ Department of Physics and Center for Computational Science, Boston University, Boston, MA 02215, USA \\ ${ }^{3}$ Higgs Centre for Theoretical Physics, University of Edinburgh, Edinburgh, EH9 3FD, UK
}

\begin{abstract}
Mass-split systems based on a conformal infrared fixed point provide a lowenergy effective description of beyond the standard model systems with large scale separation. We report results of exploratory investigations with four light and eight heavy flavors using staggered fermions, and up to five different values for the light flavor mass, five different heavy flavor masses, and two values of the bare gauge coupling.
\end{abstract}

\section{Introduction}

We focus on "beyond the standard model" (BSM) theories based on strong dynamics. These theories assume the existence of a strongly coupled system of gauge fields and fermions with a broken chiral symmetry: three Goldstone bosons provide the longitudinal components of the $W$ 's and $Z$, while the Higgs emerges either as a pseudo-Goldstone boson or as a dilaton. Experiment demands that a viable theory accommodates a large separation between the scale of electroweak symmetry breaking and the scale of excitations in the new strongly coupled sector. This is achieved by formulating the strongly coupled theory in such a way that it lies within, or close to, the conformal window, i.e. the range of parameters for which a theory has an infrared fixed point (IRFP) but is still asymptotically free (see e.g. [1-5]). Now, while with some gauge groups a theory can be inside the conformal window with few fermions, in other cases, and most notably with the SU(3) gauge group, a large number of fermions is required to get a model with an IRFP. In this latter case only a small subset of the fermions can have vanishing mass: the other fermions must be given a mass to avoid the occurrence of way too many Goldstone bosons. Thus the theory becomes a "mass broken conformal theory", that is a theory which would have an IRFP with all fermions massless, but which is kept away from the IRFP by giving mass to a subset of its fermions. Purpose of this paper is to study the properties of such "mass broken conformal theories". We will proceed by presenting first some unproven, but plausible theoretical considerations based on non-perturbative Wilsonian renormalization group arguments, following them with the illustration of numerical results which appear to validate our assumptions. The conclusion will be that "mass broken conformal theories" have remarkable properties of universality, not unlike QCD where with massless quarks the theory is independent of any coupling constant.

\footnotetext{
${ }^{\star}$ Speaker, e-mail: rebbi@bu.edu

${ }^{\star}$ Present address: Department of Physics, University of Colorado, Boulder, CO 80309, USA
} 


\section{Theoretical Considerations}

In this section we will formulate some theoretical expectations. We consider an SU(3) lattice gauge theory with $N_{f}$ identical fermions. We take the lattice to be of infinite extent, we denote by $\beta$, in the standard manner, a parameter proportional to the inverse gauge coupling squared, denote by am the mass of the fermions in lattice units ${ }^{1}$, and assume that the fermions have been discretized in a manner which preserves chiral symmetry for $a m=0$ and avoids unwanted fermionic copies. When we mention an observable, we take this to be the actual value taken by the observable, which is in principle theoretically well defined.

For our discussion, let us take first $N_{f}=4$. The fermion mass am can be taken to zero. The theory exhibits chiral symmetry breaking. Observables such as the pseudoscalar decay constant $a F_{\pi}$, masses $a M$ etc. have well defined finite values in lattice units which depend only on $\beta$. The continuum limit is recovered by letting $\beta \rightarrow \infty$ : in this limit dimensionless ratios of observables approach their continuum values, any non-vanishing observable can be used to set the scale and all other observables are fully determined in a manner independent of any free parameter (dimensional transmutation.) We may take one or two masses, $a m_{1}, a m_{2}$, different from zero. As $\beta \rightarrow \infty$ to recover the continuum limit we must also send $a m_{1}, a m_{2} \rightarrow 0$ in a controlled manner. The spectrum of masses and other observables will depend now on the way $a m_{1}, a m_{2}$ go to zero as $\beta \rightarrow \infty$ and the ratios of observables to $F_{\pi}$ will tend to finite limits. We recover QCD with massless up and down quarks and non-vanishing strange and charm masses. Nothing new here: we described a QCD-like theory with massless up and down quarks and non-vanishing strange and charm quark masses.

Let us consider then the $\mathrm{SU}(3)$ theory with 12 fermions of mass $a m=0$. We assume that the theory is conformal. The correlations functions will exhibit non-trivial power law behavior at large distances. If we rescale lengths appropriately, the long range behavior with any two values of $\beta$ will be the same, because under renormalization group transformations the theory runs to to an IRFP, in the space of infinite couplings. (The location of the IRFP will depend on the specifics of the renormalization.) The continuum behavior of the correlation functions is recovered by going to very large lattice distances. If we are close enough to the IRFP, the lattice will approximate well the continuum also at moderate and small distances: then the short distance behavior of the correlation functions will be trivial because the theory is asymptotically free in the UV.

Assume now that we keep 4 fermions massless and give a mass am to the other 8 fermions [68]. The observables (in lattice units) will now depend on $\beta$ and am. As in the case with $N_{f}=4$, at large distances the correlation functions will exhibit exponential behavior and we can measure a non-vanishing $a F_{\pi}$ as well as non-vanishing masses and other observables. In a renormalization transformation, in the space of infinite couplings, the system will move away from the IRFP with $a m=0$ because $a m$ is a relevant parameter. To recover the continuum limit with $a F_{\pi} \rightarrow 0$ we must let $a m$ approach its fixed-point value $a m=0$, but, and here is the crucial point, contrary to the case of the theory with $N_{f}=4$, now we do not need to let $\beta \rightarrow \infty$, since, as am $\rightarrow 0$, the theory will flow closer and closer to the IRFP no matter what the original $\beta$ is. If we take am sufficiently small, the ratios of observables, whether built out of the massless fermions, massive fermions or both, and in particular the ratios of observables to $F_{\pi}$, will be independent of $a m$ and $\beta$. If we give two different masses, $a m_{1}, a m_{2}$, to the massive fermions, or if we give a small mass $a m_{\ell}$ to the massless fermions, hyperscaling arguments $[8,9]$ show that the ratios of observables to $F_{\pi}$ or among themselves only depend on the ratios of the bare Lagrangian masses.

\footnotetext{
${ }^{1}$ In this paper we follow the convention of using symbols such as $m, M, F_{\pi}$ to denote dimensionful quantities. Correspondingly the mass of the fermions in lattice units must be denoted by am, $a$ being the lattice spacing, although in absence of scale setting, neither $a$ nor $m$ are separately defined.
} 
In conclusion, we can take $F_{\pi}$ to set the scale, and then the continuum theory is fully defined by the ratio of Lagrangian masses. In particular, if we only have a common mass parameter am for the 8 massive fermions, while the 4 light fermions are kept at zero mass, then the theory is fully defined without any free parameter. In this case am plays a role similar to $\beta$ for massless QCD.

\section{Numerical Results}

In this section we illustrate numerical results which appear to validate the theoretical arguments presented above. We simulated a theory [6-8] with:

- one staggered field (=4 flavors) with light mass $m_{\ell}$ plus two staggered fields ( $=8$ flavors) with heavy mass $m_{h}$. The simulations have been done done with $a m_{\ell}=0.003,0.005,0.010,0.015,0.025,0.035$, $a m_{h}=0.050,0.060,0.080,0.100$;

- a fundamental-adjoint gauge action with $\beta=4.0, \beta_{a}=-\beta / 4$ [10, 11], and nHYP smeared staggered fermions [12,13];

- lattice sizes mostly $24^{3} \times 48$ and $32^{3} \times 64$, but also $16^{3} \times 32$ (exploratory), $36^{3} \times 64$ and $48^{3} \times 96$.

- We also simulated a system with $\beta=4.4, a m_{h}=0.070, a m_{\ell}=0.009,0,013125,0.0175,0.0245$, on a $32^{3} \times 64$ lattice.

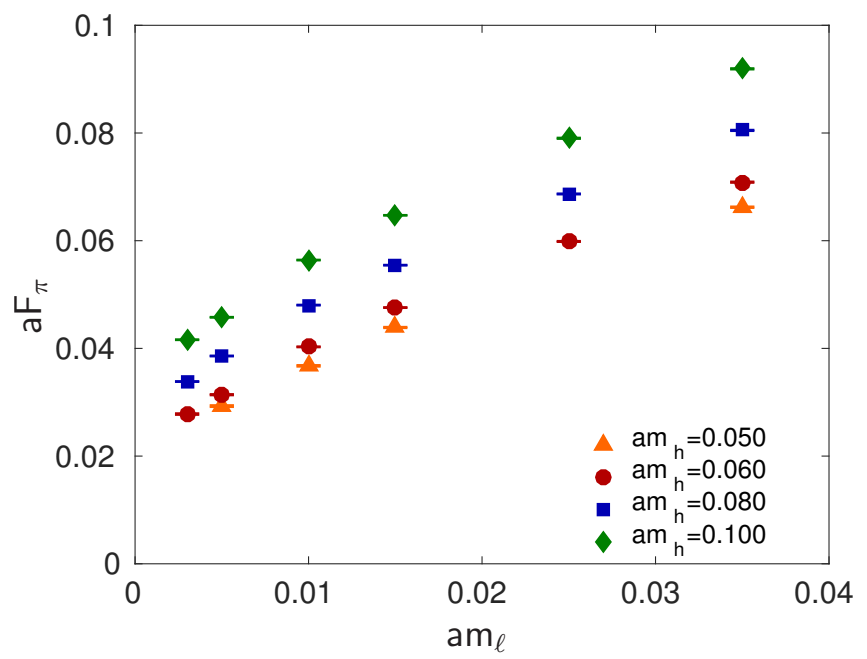

Figure 1. The pseudoscalar decay constant in lattice units.

In Figure 1 we present the value of the pseudoscalar decay constant, $a F_{\pi}$ in lattice units. $a F_{\pi}$ is seen to decrease as $a m_{h}$ decreases, following the expectation that it should go to zero as $a m_{h}$ approaches the fixed-point value $a m_{h}=0$.

Our light spectrum results for $\beta=4.0$ are summarized in the four panels of Fig 2. (In this figure, as well as in the following figures, we use the symbols $\pi, \rho, \ldots$ as a short-hand for pseudoscalar, vector etc., referring to the particle state with the same quantum numbers as in QCD.) We plot ratios of hadron masses $M_{H}$ over $F_{\pi}$, and as functions of $m_{\ell} / m_{h}$, in order to remove the scale dependence. We note that $M_{\pi} / F_{\pi}$ decreases towards zero for $m_{\ell} / m_{h} \rightarrow 0$, giving evidence of chiral symmetry breaking. We also observe that the $0^{++}$is light, with $M_{0^{++}}<M_{\rho}$, tracking the pion. 

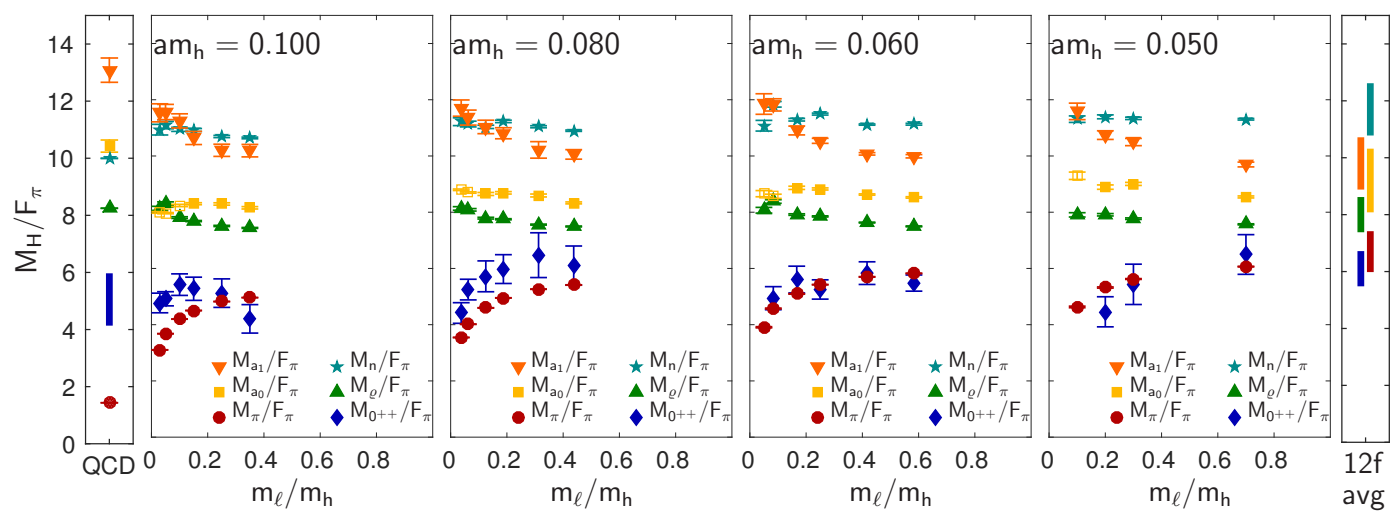

Figure 2. Ratios of the masses of $\pi, \rho, a_{0}, a_{1}, n$, and of the $0^{++}$over $F_{\pi}$. The left panel shows results for QCD taken from the PDG [14], the right panel averages for a (mass deformed) 12 flavor theory [11, 15-17].

In Figure 3 we superimpose the data obtained with different values of $m_{h}$. Hyperscaling arguments $[8,9]$ indicate that in the vicinity of the IRFP all scale independent quantities will be only a function of $m_{\ell} / m_{h}$, with no separate dependence on $m_{h}$. The way in which the data appear to line-up in Fig. 3 provides strong evidence for hyperscaling.
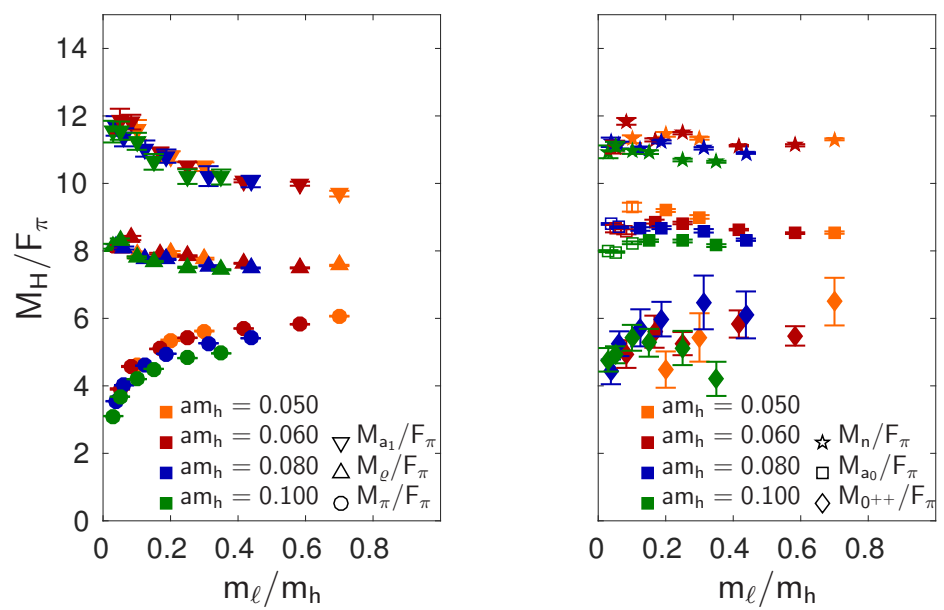

Figure 3. Hyperscaling: light hadron masses. Data for different $m_{h}$ are superimposed.

We also calculated the spectrum of masses for states made of heavy fermions. The spectra of light-light and heavy-heavy meson composites is illustrated in the the three panels of Fig. 4. The heavy-heavy spectrum also gives clear evidence of hyperscaling.

The upward bend in the heavy-heavy spectrum is in part due to the fact that light-light $F_{\pi}$ shows a marked decrease for $m_{\ell} / m_{h} \rightarrow 0$. 


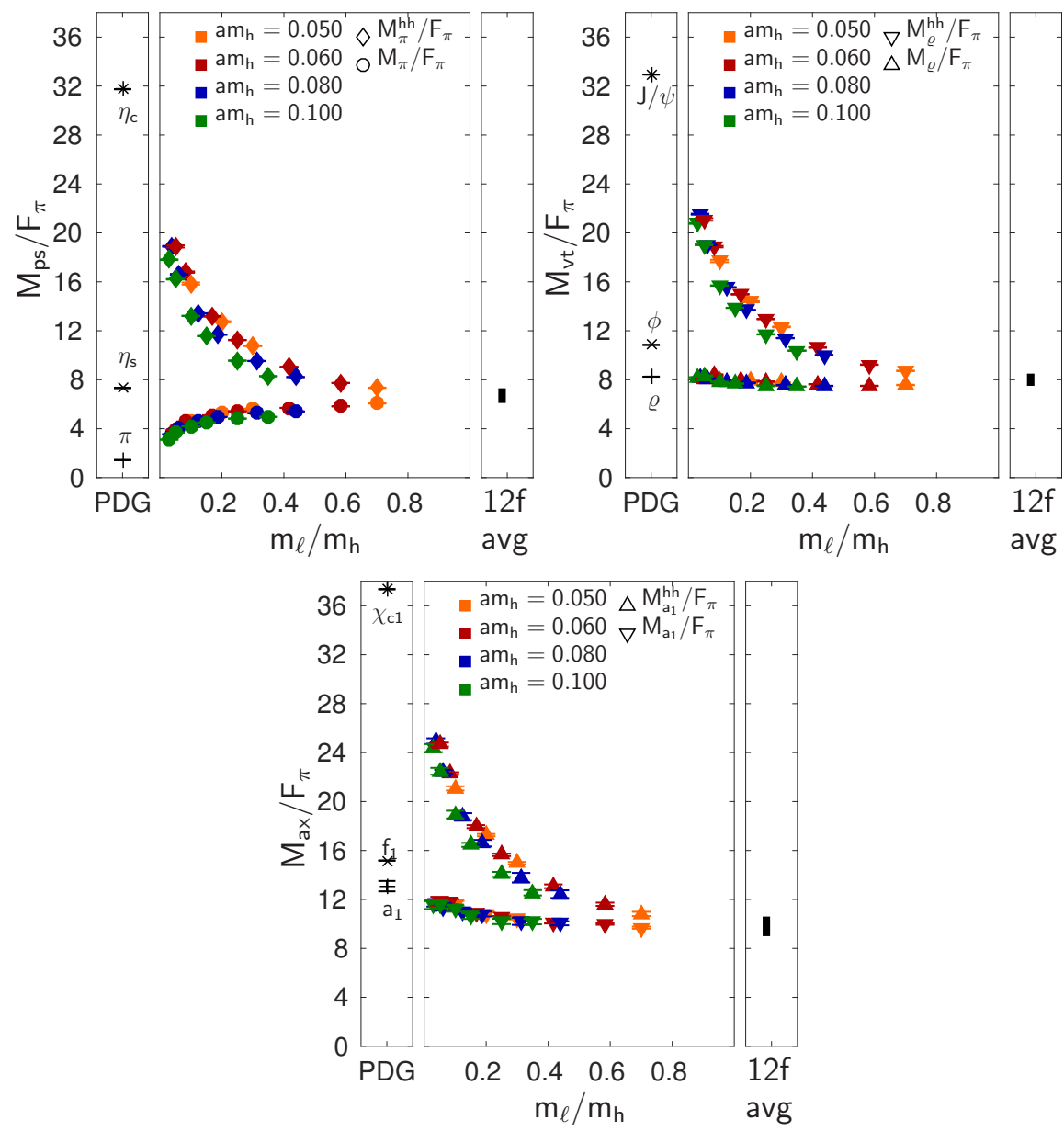

Figure 4. Ratios of the masses of $\pi, \rho, a_{1}$ over $F_{\pi}$ for light-light and heavy-heavy composites. The left panels shows result for QCD [14] with the (unphysical) $\eta_{s}$ taken from [18], the right panels for a (mass deformed) 12 flavor theory [11, 15-17].

In Figure 5 we show the ratio of the heavy-heavy $\pi$ and $a_{1}$ masses over the mass of light-light and heavy-heavy $\rho$. Once again the upward bend in the left panel can be attributed to the decrease of the light-light $M_{\rho}$ in the denominator. Ratios against heavy-heavy $M_{\rho}$ show little variation.

Figure 6 presents more detailed evidence for the fact that chiral symmetry is broken. The left panel shows that as the ratio $m_{\ell} / m_{h}$ goes to zero, $M_{\pi}$ tends to zero while $F_{\pi}$ tends to a finite limit, a clear indication of chiral symmetry breaking. In order to display physical values for $F_{\pi}$, as opposed to $a F_{\pi}$ (in lattice units), we multiply $F_{\pi}$ by a scale, denoted by $a_{\star}$, which represents the lattice spacing for $\beta=4.0, a m_{\ell}=0.003, a m_{h}=0.080$ as determined through the Wilson flow. The values in lattice units, $a M_{\pi}, a F_{\pi}$ at all other values of $a m_{\ell}, a m_{h}$ are converted to the values in Fig. 6 multiplying them by $a_{\star} / a$ as determined by the Wilson flow. The sharp increase of $M_{\rho} / M_{\pi}$ for $m_{\ell} / m_{h} \rightarrow 0$, which is seen in the right panel of Fig. 6, is more evidence of chiral symmetry breaking. 

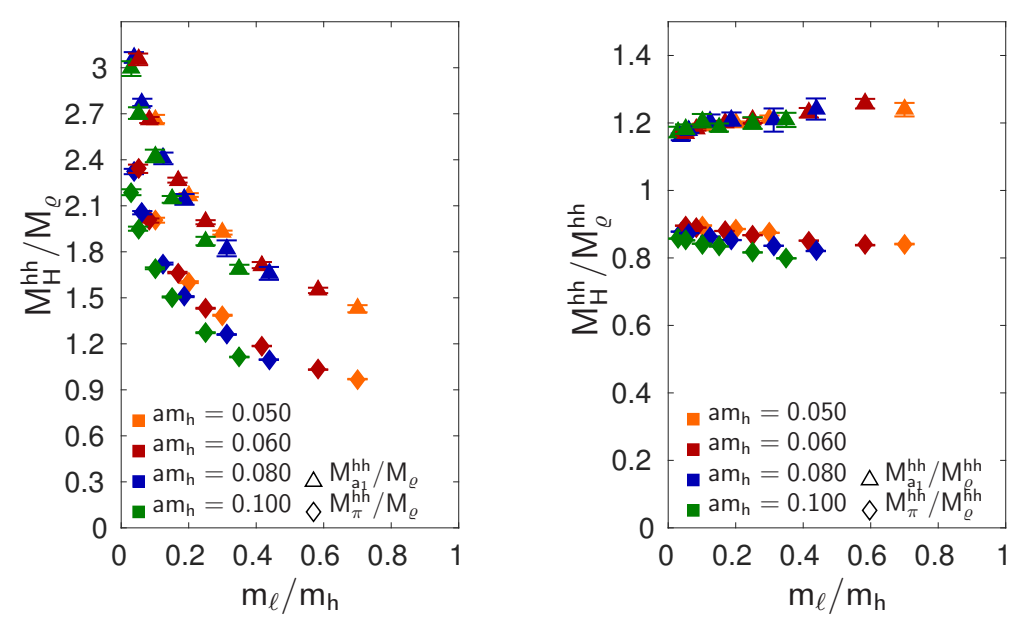

Figure 5. Ratios of the heavy-heavy $\pi$ and $a_{1}$ masses over the mass of the light-light $\rho$ (left panel) and of the heavy-heavy $\rho$.
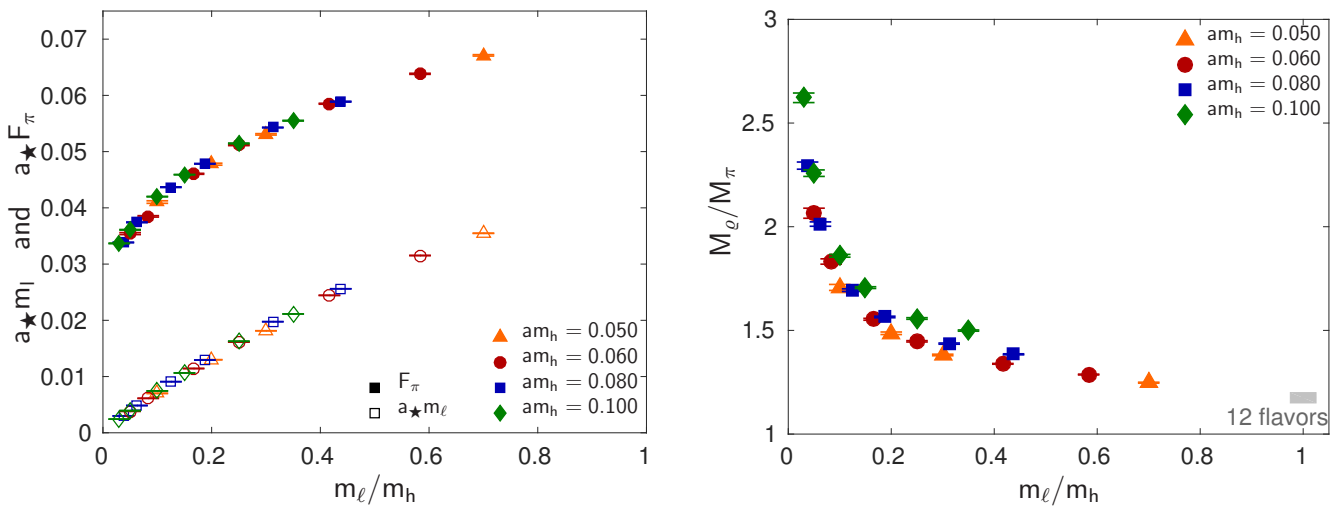

Figure 6. Right panel: $F_{\pi}$ and the light fermion mass $m_{\ell}$ as functions of the ratio $m_{\ell} / m_{\pi}$. For this graph the scale is based on the Wilson flow. Left panel: Ratio of the $\rho$ mass over the $\pi$ mass.

In Section 2 we stated that in the neighborhood of the IRFP the coupling constant $\beta$ is an irrelevant parameter. In order to verify that this is indeed the case we performed additional simulations with $\beta=4.4, a m_{h}=0.070$. If $\beta$ is an irrelevant parameter, then the ratios of physical quantities should be largely independent of its value. In Figure 7 we plot the results for $M_{\pi}, M_{\rho}, M_{a_{1}}$, in units of $F_{\pi}$, obtained from all our simulations. We already observed how the data corresponding to different $m_{h}$ at $\beta=4.0$ fall on top of each other, confirming hyperscaling. From Figure 7 we see that also the data obtained with $\beta=4.4$ follow the same pattern. While the small deviations from perfect alignment can be explained by scaling violations, finite size effects, and other systematic factors, the way in which all the data appear to line up, showing a common dependence on $m_{\ell} / m_{h}$, is impressive and appear to validate the theoretical expectations expounded in Sect. 2. 

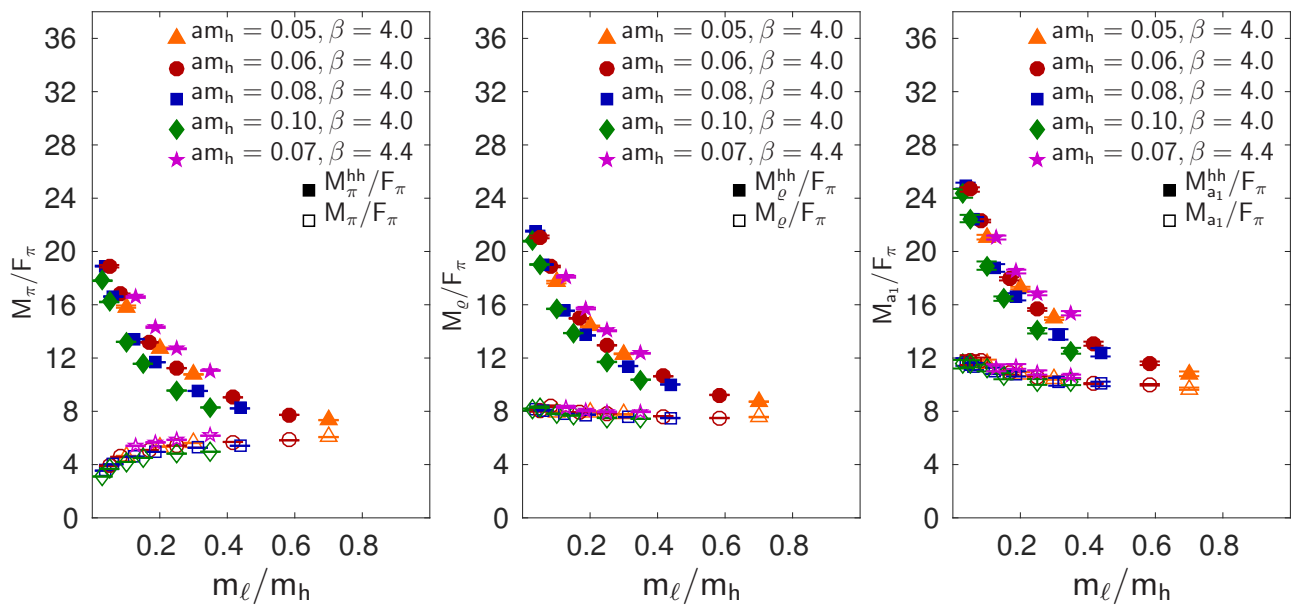

Figure 7. The coupling constant $\beta$ is an irrelevant parameter: data obtained for the light-light and heavy-heavy masses of $\pi, \rho$ and $a_{1}$ with $\beta=4.0, a m_{h}=0.100,0.080,0.060,0.050$ and $\beta=4.4, a m_{h}=0.070$ are superimposed.

\section{Conclusions and Outlook}

Our results give evidence for hyperscaling in the heavy fermion masses and for the irrelevance of the gauge coupling: for sufficiently small $m_{h}$, ratios of physical quantities will depend only on $m_{\ell} / m_{h}$ and will be independent of $\beta$. The continuum limit would be reached for $m_{\ell}, m_{h} \rightarrow 0$ with $m_{\ell} / m_{h}$ kept fixed. In particular, in the chiral limit $m_{\ell}=0, m_{h}$ would only serve to set the scale.

There is some analogy between $m_{h}$ in our model and the bare coupling constant $g$ in QCD: near the fixed-point the physical values of masses and other observables do not depend on them. In principle, in the chiral limit, the theory built in the neighborhood of the IR fixed-point would be a self-consistent, parameter free theory, very much like QCD with massless quarks.

However, even if a theory built on the IR fixed-point were self-consistent, like QCD it would have to be embedded into a more general BSM framework. Then, on various phenomenological grounds (scaling dimensions of the condensate, of baryonic operators etc.), it can be argued that the theory should be strongly coupled: thus, still within the conformal window, but as close as possible to its beginning.

There is some evidence that an SU(3) theory with 10 massless flavors may be conformal [19, 20]. This makes it quite valuable to perform an investigation, similar to the one presented in these proceedings, but with 4 massless and 6 massive fermions. Because of computational and, to some extent, theoretical difficulties [21], however, a simulation with 10 flavors could not avail itself of the simplifications brought about by staggered fermions. Moreover the need of preserving a clearly defined chiral limit practically forces one to using the much more computationally demanding domain-wall discretization. This is currently planned by the Lattice Strong Dynamics collaboration (http://lsd.physics.yale.edu/). Hopefully, the efforts of the LSD collaboration and/or some other group endowed with sufficient computational resources will clarify, in a not too distant future, the properties of the SU(3) four-plus-six fermion system. 


\section{Acknowledgments}

The authors thank their colleagues in the LSD Collaboration for fruitful and inspiring discussions. Computations for this work were carried out in part on facilities of the USQCD Collaboration, which are funded by the Office of Science of the U.S. Department of Energy, on computers at the MGHPCC, in part funded by the National Science Foundation (award OCI-1229059), and on computers allocated under the NSF Xsede program to the project TG-PHY120002. We thank Boston University, Fermilab, the NSF and the U.S. DOE for providing the facilities essential for the completion of this work. A.H. acknowledges support by DOE grant DE-SC0010005 and C.R. by DOE grant DE-SC0015845. This project has received funding from the European Union's Horizon 2020 research and innovation programme under the Marie Skłodowska-Curie grant agreement No 659322.

\section{References}

[1] M.A. Luty, T. Okui, JHEP 09, 070 (2006), hep-ph/0409274

[2] D.D. Dietrich, F. Sannino, Phys. Rev. D75, 085018 (2007), hep-ph/0611341

[3] L. Vecchi (2015), 1506.00623

[4] G. Ferretti, D. Karateev, JHEP 03, 077 (2014), 1312 . 5330

[5] T. Ma, G. Cacciapaglia, JHEP 03, 211 (2016), 1508.07014

[6] R.C. Brower, A. Hasenfratz, C. Rebbi, E. Weinberg, O. Witzel, J. Exp. Theor. Phys. 120, 423 (2015), 1410.4091

[7] R.C. Brower, A. Hasenfratz, C. Rebbi, E. Weinberg, O. Witzel, Phys. Rev. D93, 075028 (2016), 1512.02576

[8] A. Hasenfratz, C. Rebbi, O. Witzel, Phys. Lett. B773, 86 (2017), 1609.01401

[9] A. Hasenfratz, C. Rebbi, O. Witzel, PoS LATTICE2016, 226 (2016), 1611.07427

[10] A. Cheng, A. Hasenfratz, G. Petropoulos, D. Schaich, PoS LATTICE2013, 088 (2013), 1311.1287

[11] A. Cheng, A. Hasenfratz, Y. Liu, G. Petropoulos, D. Schaich, Phys.Rev. D90, 014509 (2014), 1401.0195

[12] A. Hasenfratz, F. Knechtli, Phys. Rev. D64, 034504 (2001), hep-lat/0103029

[13] A. Hasenfratz, R. Hoffmann, S. Schaefer, JHEP 0705, 029 (2007), hep-lat/0702028

[14] K.A. Olive et al. (Particle Data Group), Chin. Phys. C38, 090001 (2014)

[15] Y. Aoki, T. Aoyama, M. Kurachi, T. Maskawa, K.i. Nagai, H. Ohki, A. Shibata, K. Yamawaki, T. Yamazaki (LatKMI), Phys. Rev. D86, 054506 (2012), 1207.3060

[16] Z. Fodor, K. Holland, J. Kuti, D. Nogradi, C. Schroeder, K. Holland, J. Kuti, D. Nogradi, C. Schroeder, Phys. Lett. B703, 348 (2011), 1104 . 3124

[17] Y. Aoki, T. Aoyama, M. Kurachi, T. Maskawa, K.i. Nagai, H. Ohki, E. Rinaldi, A. Shibata, K. Yamawaki, T. Yamazaki (LatKMI), Phys. Rev. Lett. 111, 162001 (2013), 1305.6006

[18] R.J. Dowdall, C.T.H. Davies, G.P. Lepage, C. McNeile, Phys. Rev. D88, 074504 (2013), 1303.1670

[19] T.W. Chiu (2016), 1603.08854

[20] T.W. Chiu, PoS LATTICE2016, 228 (2017)

[21] A. Hasenfratz, C. Rebbi, O. Witzel, talk given at the 2017 Lattice Symposium (2017), 1708.03385 